\title{
Local Polyakov loop domains and their fractality
}

\author{
Hans-Peter Schadler* \\ Institut für Physik, Karl-Franzens Universität Graz, 8010 Graz, Austria \\ E-mail: hpseabyle.org
}

\section{Gergely Endrődi}

Institut für Theoretische Physik, Universität Regensburg, 94040 Regensburg, Germany

E-mail: gergely • endrodiaphysik.uni-regensburg.de

\section{Christof Gattringer}

Institut für Physik, Karl-Franzens Universität Graz, 8010 Graz, Austria

E-mail: christof.gattringer@uni-graz.at

\begin{abstract}
We discuss properties of local Polyakov loops in the deconfinement transition of SU(3) lattice gauge theory at finite temperature using the fixed scale approach. In particular we study spatial clusters where local Polyakov loops have phases near the same center elements of the gauge group. We present results for various properties of the center clusters, e.g., their percolation probability or their fractality and discuss the physical implications for temperatures below and above the phase transition.
\end{abstract}

31st International Symposium on Lattice Field Theory - LATTICE 2013

July 29 - August 3, 2013

Mainz, Germany

${ }^{*}$ Speaker. 


\section{Introduction}

In recent years various experiments like the Large Hadron Collider at CERN or the Relativistic Heavy Ion Collider in Brookhaven, USA, have started to collect data and to analyze properties of the quark gluon plasma (QGP) such as its viscosity or opacity. Understanding the high temperature phase of matter is of fundamental importance for our deeper understanding of quantum chromodynamics (QCD). So far many properties of the QGP have been measured but for many of them we still lack a concluding theoretical explanation.

In recent years there have been attempts to describe phenomenological properties of the QGP [1] by using the old idea of center domains [2]. These center domains are related to the static quark potential as they are defined by the phases of the Polyakov loop. Used in the description of the QGP they may be able to explain some of the phenomena observed in heavy ion collisions.

On the lattice one has direct access to the characteristic gauge configurations which allows one to directly study the center domains defined by the local Polyakov loop. Various such studies can be found in the literature [4, 5], but so far the temperature $T=\left(a(\beta) N_{t}\right)^{-1}$ was always changed by varying the lattice constant $a(\beta)$, i.e., by changing the inverse gauge coupling $\beta$. This has the major disadvantage that various effects related to temperature, the volume and the lattice spacing $a$ were mixed. For a clean study of the properties of the center clusters one would like to disentangle these effects. This can be accomplished by using the fixed scale approach, i.e., one works at a fixed inverse gauge coupling $\beta$ (and thus fixed lattice constant $a$ ), and drives the temperature by varying the temporal lattice extent $N_{t}$.

In this preliminary study we analyze properties of the center domains of pure $\mathrm{SU}(3)$ lattice gauge theory using the Wilson plaquette action for $N_{s}^{3} \times N_{t}$ lattices of spatial sizes $N_{s}=30,40$ and $N_{s}=$ 48. The temperature is driven by varying the inverse temporal lattice extent $N_{t}=2, \ldots, 20$ and we perform calculations for three different lattice spacings $a=0.1117,0.0677$ and $0.0481 \mathrm{fm}$.

\section{General properties of the Polyakov loop}

In the continuum the local Polyakov loop at a spatial point $x$ is defined as the trace of the pathordered exponential

$$
L(x)=\operatorname{Tr} \mathscr{P} \exp \left(\int_{0}^{1 / T} A_{4}(x, t) d t\right),
$$

where $t$ is the euclidean time which is integrated between 0 and the inverse temperature $1 / T$ (we set the Boltzmann constant to $k_{B}=1$ ). On the lattice, the local Polyakov loop is given by the trace of the product over all time-like links $U_{4}(x, t)$

$$
L(x)=\operatorname{Tr} \prod_{t=1}^{N_{t}} U_{4}(x, t),
$$

which is obviously a gauge invariant object. Under a center transformation, i.e., multiplying all temporal links $U_{4}\left(x, t_{0}\right)$ on a certain time-slice $t_{0}$ with a center element $z \in\left\{1, e^{i 2 \pi / 3}, e^{-i 2 \pi / 3}\right\}$ of $\mathrm{SU}(3)$, the local Polyakov loop transforms non-trivially and picks up this center element as a factor, $L(x) \rightarrow z L(x)$. Thus it serves as an order parameter for the breaking of the center symmetry which for pure gauge theory is a symmetry of the action. Above the critical temperature $T>T_{c}$ the center 
symmetry is spontaneously broken, manifest in a non-vanishing expectation value for the local Polyakov loop $\langle L(x)\rangle \neq 0$. In the case of dynamical fermions, the fermion determinant breaks the center symmetry explicitly and the system favors the trivial center sector around $z=1$ resulting in a non-vanishing Polyakov loop expectation value already below the transition.

From previous work [5] it is known that the phase $\theta(x)$ of the Polyakov loop plays a special role (we write $L(x)=|L(x)| \exp (i \theta(x))$ ). Below the phase transition the phases $\theta(x)$ are equally distributed in all center sectors with peaks of equal hight at $\theta(x)=0, \pm 2 \pi / 3$ corresponding to the center elements of $\mathrm{SU}(3)$. Averaging over the phases leads to the vanishing expectation value $\langle L(x)\rangle=0$. Above the phase transition, the behavior is different. The system will spontaneously choose a preferred center sector and the distribution of the phases $\theta(x)$ will show a peak at the corresponding center element which leads to a non-zero expectation value $\langle L(x)\rangle$.

\section{Definition of center sectors and clusters}

Based on the qualitative discussion of the properties of the local Polyakov loops $L(x)$ and its phases $\theta(x)$, we assign to every spatial point $x$ the center sector number $n(x)$ according to the following prescription,

$$
n(x)=\left\{\begin{aligned}
-1 & \text { for } \theta(x) \in[-\pi+\delta,-\pi / 3-\delta] \\
0 & \text { for } \theta(x) \in[-\pi / 3+\delta, \pi / 3-\delta], \\
+1 & \text { for } \theta(x) \in[\pi / 3+\delta, \pi-\delta]
\end{aligned}\right.
$$

with a real and non-negative parameter $f$,

$$
\delta=f \frac{\pi}{3}, \quad f \in[0,1],
$$

which we refer to as the cut parameter. The role of the parameter is to allow for removing sites $x$ from the cluster analysis where the local Polyakov loops $L(x)$ do not clearly lean towards one of the center elements: For $f=0$ no sites are removed, while for $f \rightarrow 1$ all sites are removed, and values in between the two extrema allow a gradual removal of "undecided" sites. Fig. 1 illustrates a possible decomposition of a (2-dimensional) lattice into sites with $n(x)=0, \pm 1$ and sites that are removed with a finite value of $f$.

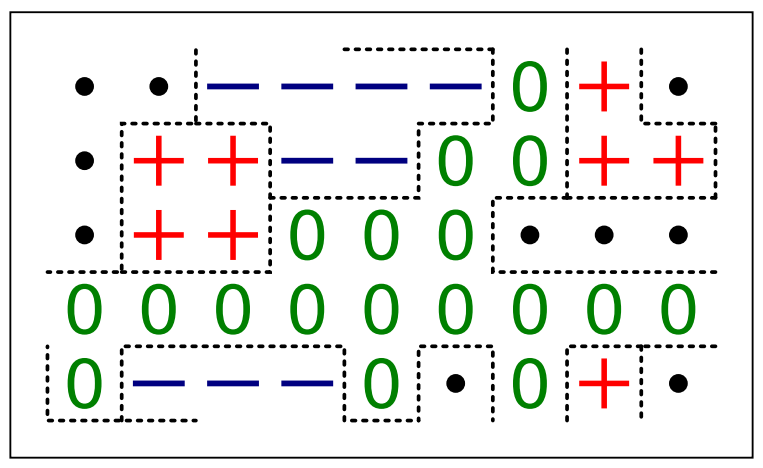

Figure 1: A possible cluster configuration with clusters in all three sectors $0, \pm 1$. The black dots correspond to spatial points which are removed for some $f>0$. Some of the clusters are connected via the periodic boundary conditions and the sector 0 (green) percolates, i.e., it extends over the whole lattice in all directions. 

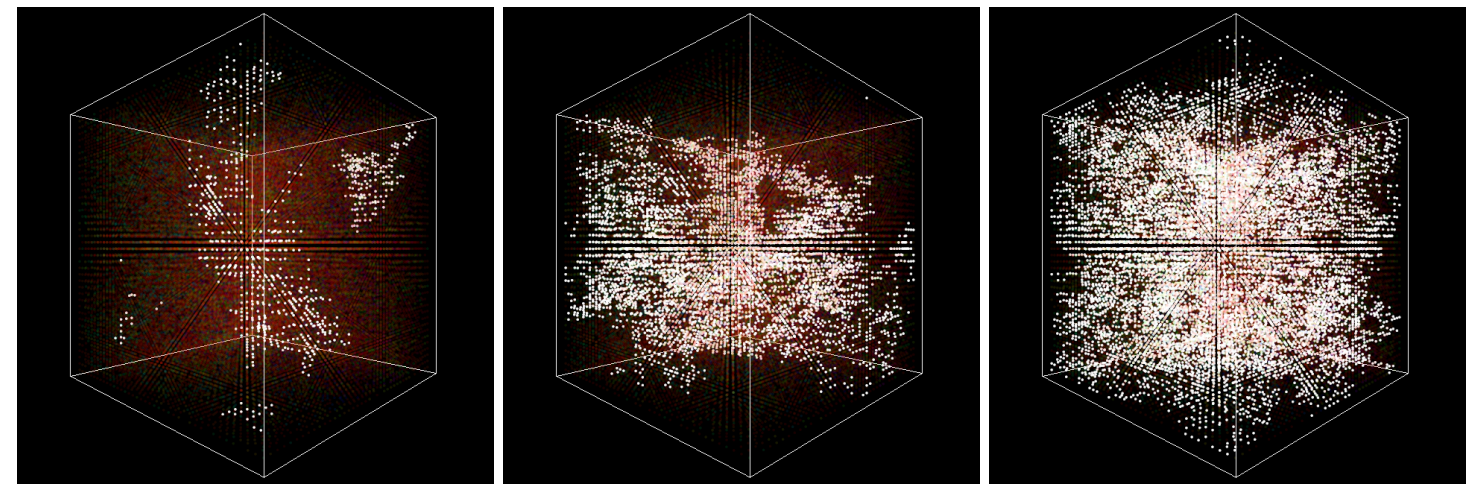

Figure 2: The largest center cluster on a $40^{3} \times N_{t}$ lattice for temperatures $T / T_{c}=0.90,0.99,1.10$ (from left to right) at a cut parameter $f=0.3$.

Center clusters are now defined in the following way: Two neighboring spatial points $x$ and $y=$ $x \pm \hat{\mu}$ belong to the same cluster if $n(x)=n(y)$, i.e., if they are near the same center element. In Fig. 1 we show a possible schematic configuration on a 2-D slice of the lattice and in Fig. 2 we show the largest cluster on a $N_{s}=40$ lattice at three different temperatures for a cut parameter of $f=0.3$. It is obvious that when the temperature increases above $T_{c}$, the largest cluster dramatically increases in size and starts to percolate. Exactly these changes of the center clusters near $T_{c}$ are at the focus of this study.

Before we discuss properties of the clusters, we need to illuminate a little bit the role of the cut parameter $f$. We start with an excursion to percolation theory and recall some fundamental properties: In random percolation theory the threshold for site percolation on a simple cubic 3-D lattice is $p=p_{c}=0.3116$ [6], where $p$ is the occupation probability for a lattice site, i.e., if the occupation probability $p$ is larger than $p_{c}$ we expect to find a percolating cluster. If we assume random distribution of the values $n(x) \in\{0, \pm 1\}$ and use a cut parameter $f=0$ (no sites removed), we get for every center sector an occupation probability of $p=1 / 3>0.3116=p_{c}$, and thus expect that without cut there is a percolating cluster in each of the three center sectors. This is confirmed in Fig. 3 where we show the number of percolating clusters on a $N_{s}=48$ lattice as a function of the

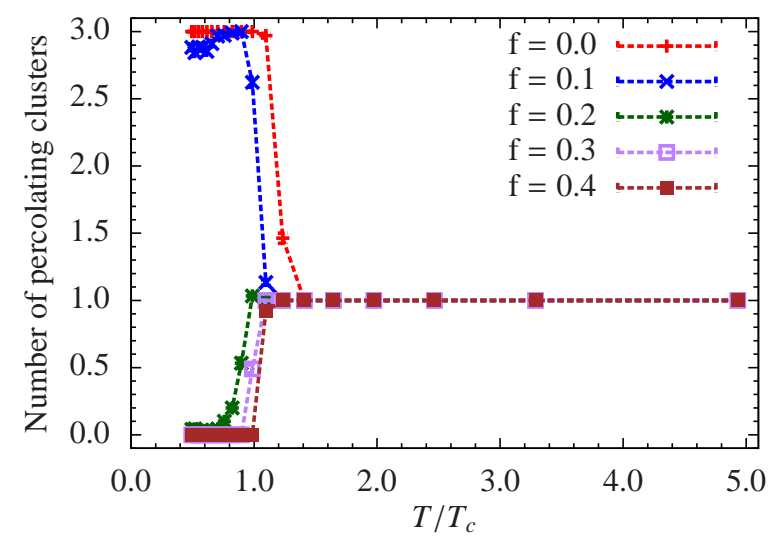

Figure 3: Number of percolating clusters as a function of the temperature for different cut parameters $f$. We show the results for the $N_{s}=48, a=0.0677 \mathrm{fm}$ ensemble. 
temperature $T$ for different values of the cut parameter $f$. We observe the following: For $f=0$ we indeed find three percolating clusters for $T<T_{c}$, one for every center sector. For temperatures $T>T_{c}$ one of the center sectors will become dominant and the number of percolating clusters drops to one. For sufficiently large $f$ the picture is reversed, and below $T_{c}$ there is no percolating cluster while for $T>T_{c}$ a single percolating cluster emerges (compare Fig. 2).

\section{Cluster weight}

Simple to calculate, but nevertheless important, are observables related to the number of sites which belong to a cluster, i.e., the cluster weight. We study two different definitions: In Fig. 4 (1.h.s.) we show the average weight of the largest cluster, i.e., the number of lattice sites which belong to this cluster, normalized by the 3-volume $V=N_{s}^{3}$ for three different spatial volumes. The cut parameter was chosen to be $f=0.3$ and we work at $a=0.0677 \mathrm{fm}$. Below the critical temperature the cluster weight does not depend on the temperature but shows a plateau. Furthermore, these plateaus have different values for the three different volumes, which indicates that the cluster weight is only weakly dependent on the volume below $T_{c}$ and the visible volume dependence in Fig. 4 comes solely from the normalization. At the critical temperature the weight of the largest cluster starts to increase as the cluster starts to percolate. From the different volumes we observe the usual rounding which is related to finite volume effects. Above the critical temperature $T>T_{c}$ there is perfect agreement between the different volumes and for high $T$ the weight tends to $\left\langle W_{L}\right\rangle / V=1$. Also an interesting quantity, which serves as a susceptibility-like observable, is the average weight $W_{n p}$ of the non-percolating clusters. We define it as (see, e.g., $[3,4]$ )

$$
w_{s}=\frac{n_{s} s}{\sum_{s^{\prime}} n_{s^{\prime}} s^{\prime}} \quad, \quad W_{n p}=\sum_{s} w_{s} s=\frac{\sum_{s} n_{s} s^{2}}{\sum_{s^{\prime}} n_{s^{\prime}} s^{\prime}},
$$

where $s$ is the size of the cluster and $n_{s}$ is the number of clusters of size $s$. With this definition, $w_{s}$ is the probability that an occupied site belongs to a cluster of size $s$. Using this, we define the average weight $W_{n p}$ of the non-percolating clusters as given in the second equality of (4.1) where all sums run over all non-percolating clusters.
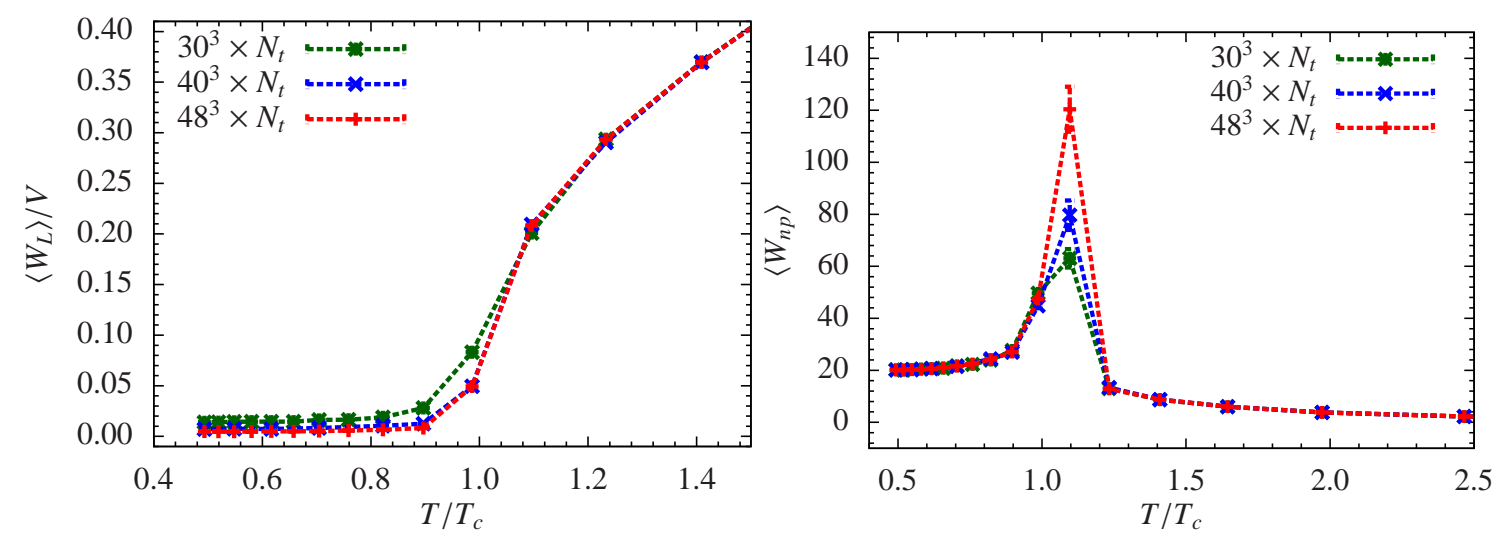

Figure 4: Comparison of the weight of the largest cluster (1.h.s.) and of the average non-percolating clusters (r.h.s.) for three different lattice volumes as a function of the temperature $T$. We show the results for the $a=0.0677 \mathrm{fm}$ ensemble with a cut parameter $f=0.3$. 
We study the expectation value $\left\langle W_{n p}\right\rangle$ as a function of the temperature, again for three different volumes (Fig. 4, r.h.s.), and find a peak at the critical temperature $T=T_{c}$. Furthermore, also the expected scaling of the peak height with the lattice volume can be observed.

\section{Fractal dimension of the center clusters}

The last property that we want to discuss is the fractal dimension of the largest cluster. We determine the fractal dimension via the box counting method. In this method, one counts the number of boxes $N(s)$ of size $s$ needed to cover the whole cluster. In the limit of small $s$ we expect to find a behavior given by

$$
N(s)=C s^{-D_{b o x}} .
$$

In Fig. 5 we plot the box counting dimension, obtained by a fit of the data to Eq. (5.1), for our $a=0.0677 \mathrm{fm}$ ensemble as a function of the temperature for three different spatial volumes. The fractal dimension changes drastically with the temperature: While below $T_{c}$ the dimension is around $D_{b o x} \approx 1.6$, it shows a drastic increase at the critical temperature and approaches $D_{b o x}=3.0$ already slightly above $T_{c}$. The $N_{s}=30$ ensemble shows strong finite size effects below $T_{c}$ which makes a reliable determination of $D_{b o x}$ challenging. For the $N_{s}=40$ ensemble, we only observe a mild rounding at $T=T_{c}$.

\section{Conclusion and final remarks}

We start our concluding words with a comparison to percolation theory. The analog of the temperature in percolation theory is the occupation probability $p$. Due to the dominance of a single percolating cluster above $T_{c}$, the occupation probability for the corresponding sector becomes larger with increasing temperature and at some point reaches the critical value $p_{c}$ where a cluster of the

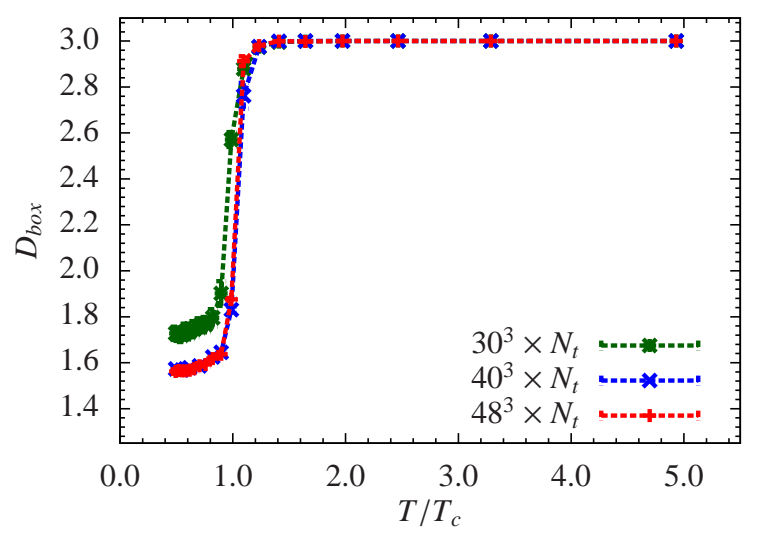

Figure 5: Box counting dimension $D_{b o x}$ as a function of the temperature for different spatial volumes. We use our $a=0.0677 \mathrm{fm}$ ensemble. The cut parameter $f$ is tuned such that the physical radius of the largest cluster at the lowest temperature is $R_{\text {phys }}=0.5 \mathrm{fm}$. For $N_{s}=40$ we observe the usual rounding effect around $T_{c}$, while the $N_{s}=30$ results are plagued by more severe finite size effects which affect the quality of the fits and the resulting fractal dimension below $T_{c}$. 
dominant sector starts to percolate. The change of the temperature therefore corresponds to changing the occupation probability in favor of one sector which is spontaneously chosen by the system in the spontaneous breaking of the center symmetry. This behavior can also be observed if one sets the cut parameter to $f=0$. The corresponding transition would then be from three equally distributed sectors with $p_{i}>p_{c}, i=1,2,3$, i.e., each with a percolating cluster, down to only one dominating sector with, for example, $p_{1,2}<p_{c}$ and $p_{3} \geq p_{c}$ (compare Fig. 3).

The analysis of the weight and the fractal dimension of the largest cluster show that the clusters are small and highly fractal objects with $D_{b o x} \approx 1.6$ below the critical temperature $T<T_{c}$. At the critical temperature the fractal dimension quickly jumps to $D_{b o x}=3.0$, while the weight of the largest cluster starts to increase steadily towards $\left\langle W_{L}\right\rangle / V=1$.

We remark that the cut parameter $f$ can be related to a physical quantity, the radius of the largest cluster at $T \ll T_{c}$, which changes as a function of $f$. The radius of the largest cluster in physical units for some temperature $T<T_{c}$ can then be adjusted to some fixed value, e.g., to $0.5 \mathrm{fm}$, by adjusting $f$. Thus by suitably adjusting $f$ we can set the scale and use this procedure to compare results from lattices with different lattice constants $a$. This procedure and other properties of the center clusters will be discussed in detail in our upcoming publication [7].

\section{Acknowledgments}

We thank S. Borsányi, J. Danzer, M. Dirnberger, A. Maas, B. Müller and A. Schäfer for interesting discussions. In addition, the speaker wants to thank T. Kloiber, C. Lang and A. Schmidt. This work is partly supported by DFG TR55, "Hadron Properties from Lattice QCD" and by the Austrian Science Fund FWF Grant. Nr. I 1452-N27. H.-P. Schadler is funded by the FWF DK W1203 "Hadrons in Vacuum, Nuclei and Stars" and a research grant from the Province of Styria. G. Endrődi acknowledges support from the EU (ITN STRONGnet 238353).

\section{References}

[1] M. Asakawa, S. A. Bass, B. Müller, Phys. Rev. Lett. 110, 202301 (2013).

[2] T. Bhattacharya, A. Gocksch, C. Korthals Altes, R. D. Pisarski, Phys. Rev. Lett. 66, 998 (1991); Nucl. Phys. B 383, 497 (1992). J. Boorstein, D. Kutasov, Phys. Rev. D 51 (1995) 7111.

[3] D. Stauffer, A. Aharony, Introduction To Percolation Theory, Taylor \& Francis 1994.

[4] S. Fortunato, hep-lat/0012006 (2000).

[5] C. Gattringer, Phys. Lett. B 690 (2010) 179. J. Danzer, C. Gattringer, S. Borsanyi, Z. Fodor, PoS LATTICE2010 (2010) 176. C. Gattringer, A. Schmidt, JHEP 1101 (2011) 051. S. Borsanyi, J. Danzer, Z. Fodor, C. Gattringer, A. Schmidt, J. Phys. Conf. Ser. 312 (2011) 012005. M. Dirnberger, C. Gattringer, A. Maas, Phys. Lett. B 716 (2012) 465.

[6] N. Jan, D. Stauffer, Int. J. Mod. Phys. C 09, 341 (1998). Y. Deng, H. W. J. Blöte, Phys. Rev. E 72 , 016126 (2005). J. Wang, Z. Zhou, W. Zhang, T. M. Garoni, Y. Deng, Phys. Rev. E 87, 052107 (2013).

[7] G. Endrődi, C. Gattringer, H.-P. Schadler, in preparation. 